\title{
Leukocytoclastic Vasculitis Associated with Macrolide Antibiotics
}

\author{
Hye Yun Park ${ }^{1}$, Sung Bum Park ${ }^{1}$, Kee-Taek Jang ${ }^{2}$ and Won-Jung Koh ${ }^{1}$
}

Key words: hypersensitivity vasculitis, macrolides

(Inter Med 47: 1157-1158, 2008)

(DOI: 10.2169/internalmedicine.47.0981)

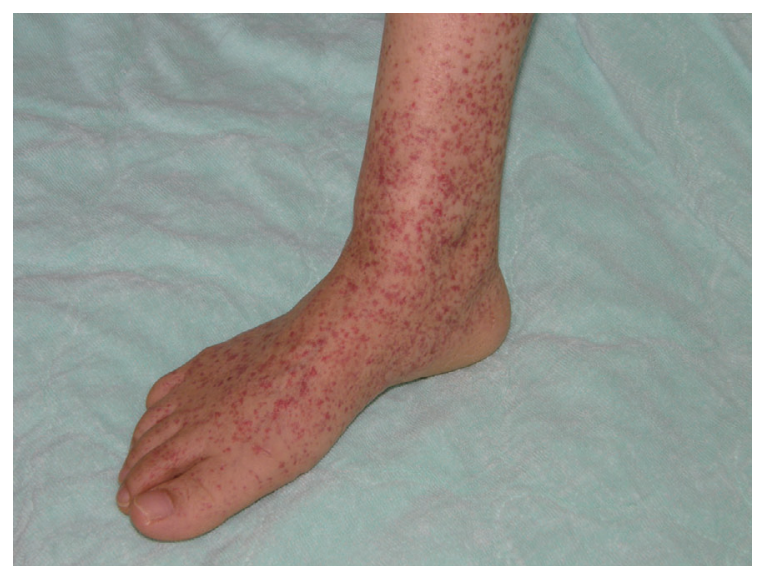

Figure 1. Purpuric nonblanching rash on the patient's foot and leg.

A 51-year-old woman was admitted to the hospital for antibiotic treatment of nontuberculous mycobacterial lung disease due to Mycobacterium abscessus. Combination antibiotic therapy, which included cefoxitin, amikacin, clarithromycin, and ciprofloxacin, was started. She had no history of allergic reactions to any drugs. Three days later, she developed a purpuric, nonblanching rash, which was palpable in sections, on both of her arms and lower legs (Fig. 1). Her platelet count and coagulation factors were normal. After the discontinuation of all antibiotics and application of topical corticosteroid therapy, the skin lesions disappeared within 6 days. A skin biopsy revealed neutrophilic vasculitis of small vessels accompanied by fibrinoid necrosis and leukocytoclasis (Fig. 2). Clarithromycin was readministrated first. The purpuric eccymotic lesions reappeared on her feet on the third day of therapy. Clarithromycin was discontinued, and the skin lesions began to fade. Three days later, azithromy-

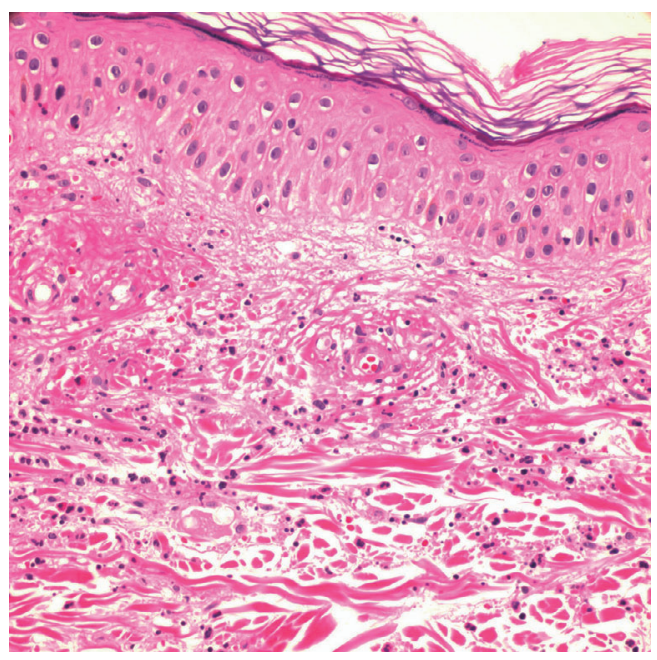

Figure 2. Neutrophilic vasculitis of small vessels accompanied by fibrinoid necrosis and leukocytoclasis (Hematoxylin and Eosin staining, $\times 400)$.

cin was administered. On the third day of azithromycin therapy, she developed a widespread purpuric rash on her arms, legs, and feet again. The skin lesions resolved after azithromycin discontinuation. Of the drugs associated with leukocytoclastic vasculitis, antibiotics are the most common agents (1). However, only a few cases of leukocytoclastic vasculitis induced by clarithromycin or azithromycin have been reported (2-4). The present case showed that both clarithromycin and azithromycin, which are the most important oral antibiotics for the long-term treatment of nontuberculous mycobacterial lung disease, may lead to leukocytoclastic vasculitis.

\footnotetext{
${ }^{1}$ Division of Pulmonary and Critical Care Medicine, Department of Medicine, Samsung Medical Center, Sungkyunkwan University School of Medicine, Seoul, Republic of Korea and ${ }^{2}$ Department of Pathology, Samsung Medical Center, Sungkyunkwan University School of Medicine, Seoul, Republic of Korea

Received for publication February 3, 2008; Accepted for publication March 26, 2008

Correspondence to Dr. Won-Jung Koh,wjkoh@skku.edu
} 
Inter Med 47: 1157-1158, 2008 DOI: 10.2169/internalmedicine.47.0981

\section{References}

1. Mathews KP. Clinical spectrum of allergic and pseudoallergic drug reactions. J Allergy Clin Immunol 74: 558-566, 1984.

2. de Vega T, Blanco S, Lopez C, Pascual E, Sanchez M, Zamarron A. Clarithromycin-induced leukocytoclastic vasculitis. Eur J Clin Microbiol Infect Dis 12: 563, 1993.

3. Gavura SR, Nusinowitz S. Leukocytoclastic vasculitis associated with clarithromycin. Ann Pharmacother 32: 543-545, 1998.

4. Odemis E, Kalyoncu M, Okten A, Yildiz K. Azithromycin-induced leukocytoclastic vasculitis. J Rheumatol 30: 2292, 2003.

(C) 2008 The Japanese Society of Internal Medicine http://www.naika.or.jp/imindex.html 